\title{
Are Narrow Line Seyfert 1s a special class of Active Galactic Nuclei?
}

\section{Valencia-S. ${ }^{* 1, \dagger}$}

E-mail: mvalencias@ph1.uni-koeln.de

\section{J. Zuther ${ }^{1}$, A. Eckart ${ }^{1,2}$, S. Smajic ${ }^{1, \dagger}$, C. Iserlohe ${ }^{1}$, M. García-Marín ${ }^{1}$, G. Busch ${ }^{1,}$, M. Vitale ${ }^{1, \dagger}$, M. Bremer ${ }^{1}$, S. Fischer ${ }^{1}$, M. Horrobin ${ }^{1}$, L. Moser ${ }^{1}$, Y.E. Rashed ${ }^{1}$, and C. Straubmeier ${ }^{1}$}

${ }^{1}$ I. Physikalisches Institut, Universität zu Köln, Zülpicher Str. 77, 50937 Köln, Germany

2 Max-Planck-Institut für Radioastronomie, Auf dem Hügel 69, 53121 Bonn, Germany

No. Due to their apparently extreme optical to X-ray properties, Narrow Line Seyfert 1s (NLSy1s) have been considered a special class of active galactic nuclei (AGN). Here, we summarize observational results from different groups to conclude that none of the characteristics that are typically used to define the NLSy1s as a distinct group - from the, nowadays called, Broad Line Seyfert 1s (BLSy1s) - is unique, nor ubiquitous of these particular sources, but shared by the whole Type 1 AGN. Historically, the NLSy1s have been distinguished from the BLSy1s by the narrow width of the broad $\mathrm{H} \beta$ emission line. The upper limit on the full width at half maximum of this line is $2000 \mathrm{~km} \mathrm{~s}^{-1}$ for NLSy1s, while in BLSy1s it can be of several thousands of $\mathrm{km} \mathrm{s}^{-1}$. However, this border has been arbitrarily set and does not correspond to the change of any physical property. All observed parameters in Type 1 AGN cover continues ranges of values, which does not allow to infer the existence of two different kind of populations with $\mathrm{FWHM}_{\mathrm{H} \beta \text {, broad }}=2000 \mathrm{~km} \mathrm{~s}^{-1}$ as dividing point. We argue that the usage of this velocity limit to define samples of NLSy1s galaxies - as it is usually done in comparative studies -, together with the well known observational biases, naturally favors the selection of sources with low black hole masses and high Eddington ratios that are hosted by blue spiral galaxies. Therefore selection biases might be responsible for the reported differences between NLSy1 and BLSy1 sources.

Nuclei of Seyfert galaxies and QSOs - Central engine \& conditions of star formation, November 6-8, 2012

Max-Planck-Insitut für Radioastronomie (MPIfR), Bonn, Germany

\footnotetext{
* Speaker.

${ }^{\dagger}$ Member of the International Max Planck Research School (IMPRS) for Astronomy and Astrophysics at the MPIfR and the Universities of Bonn and Cologne.

${ }^{\ddagger}$ Member of the Bonn-Cologne Graduate School (BCGS) of Physics and Astronomy.
} 


\section{Introduction}

Irrespective of their luminosity, active galactic nuclei (AGN) have been classified in two main types according to the possibility of having a clear view toward the central engine. Those with their symmetry axes close to the line of sight - indicated by their radio emission and/or the presence of broad lines in the spectrum - are classified as Type 1, while those at higher inclinations - suggested by the high absorption column densities in the X-rays probably originating in dusty clumps at outer regions of the accretion disk - are considered Type 2 sources. Unobscured Type 1 sources are ideal targets to study the physics of accretion onto supermassive black holes, because they allow us to reconstruct the AGN intrinsic spectral energy distributions and constrain the theoretical models (e.g., $[2,3]$ and refs. therein).

In the local universe, Type 1 AGN - constituted mostly by Seyfert $1 \mathrm{~s}$ - span a wide range of bolometric luminosities $\left(44 \lesssim \log \left(L_{\mathrm{bol}} / \mathrm{erg} \mathrm{s}^{-1}\right) \lesssim 47\right)$, black hole masses $\left(6 \lesssim \log \left(M_{\mathrm{BH}} / M_{\odot}\right) \lesssim\right.$ 9) and Eddington ratios $\left(-2 \lesssim \log \left(L_{\text {bol }} / L_{\text {Edd }}\right) \lesssim 1\right)$. These objects show broad recombination emission lines with typical widths of the order of several thousands of $\mathrm{km} \mathrm{s}^{-1}$. Osterbrock \& Pogge (1985) identified a group of AGN with "all the properties of Seyfert 1 or 1.5 galaxies, but unusually [by that time] narrow HI lines", which they called Narrow Line Seyfert 1s (NLSy1s) [25]. Later, the quantitative limit $\mathrm{FWHM}_{\mathrm{H} \beta \text {, broad }} \leq 2000 \mathrm{~km} \mathrm{~s}^{-1}$ was adopted [27]. Other properties of these objects are the relatively strong FeII emission, soft X-ray excess, steep 2-10 keV power-law (with photon index $\Gamma \gtrsim 2$ ), and short term X-ray variability. The extreme NLSy1 properties, as it has been discussed extensively in the literature, seem to be driven by the Eddington ratio - as a surrogate of the unobservable mass-accretion rate -, the black hole mass (e.g., [30, 6, 11]), and probably also by the conditions in the narrow line region (e.g., [36, 24]).

In the following, we examine some of the properties ascribed to NLSy1s in order to enlighten the discussion of whether these sources constitute a particular class of AGN, or are just normal members of the Type 1 population. Reviewing the related literature in the past couple of decades, and using published available data of thousands of Type 1 sources, we show that the properties used to characterize and define the NLSy1s as a special group of AGN neither are shared by all the members, nor they are exclusive of this kind of sources. We discuss some observational biases that affect the selection of samples, and that might also lead to conclude that NLSy1s are different from other Type 1 AGN in comparative studies. Throughout the text, we use the term Broad Line Seyfert 1s (BLSy1s) to designate Type 1 sources with broad hydrogen-line widths larger than $2000 \mathrm{~km} \mathrm{~s}^{-1}$. The term NLSy1s is employed following the designation of the authors in each contribution. In that sense, it is not uniformely defined and e.g., one finds that a source classified as NLSy1 by one group is called low- $M_{\mathrm{BH}} \mathrm{AGN}$ (with broad line emission) by another.

\section{Properties of Narrow Line Seyfert 1s (only?)}

From statistical studies of Type 1 samples, relations between different line emission parameters have been derived. NLSy1s seem to lie at one extreme of some of those relations, however a direct interpretation is not trivial essentially for two reasons: i) The sample selection. Groups of sources that span narrower parameter ranges display ambiguously correlated data, and cluster in smaller regions on the diagrams than 'control' samples with wider parameter ranges. When select- 
ing a sample, the narrower the limit imposed to the broad line width is, the smaller the ranges in e.g., $M_{\mathrm{BH}}, L_{\mathrm{bol}}$, and consequently $L_{\mathrm{bol}} / L_{\mathrm{Edd}}$ become, as we discuss later. ii) The choice of parameters to derive possible trends. Correlations between parameters that are intimately related to each other can mimic real physical trends and mislead the interpretation of the data ([33, 3]). To these caveats, one has to add the fact that data is treated in different ways by different groups. Hence, some of the correlations are not confirmed by different samples. For these reasons, we leave the discussion on correlations and trends for other ocassion, and concentrate here only on the most common properties used to describe - and to select - NLSy1s.

Hydrogen beta broad-line is narrow: FWHM $\leq 2000 \mathrm{~km} \mathrm{~s}^{-1}$. The most commonly used selection criterium for NLSy1s is the width of the broad $\mathrm{H} \beta$ line. In the literature, the $2000 \mathrm{~km} \mathrm{~s}^{-1}$ limit appears for the first time in Osterbrock \& Pogge (1987)[27] ${ }^{1}$. Although from the beginning this limit was recognized as arbitrary and unphysical [14], it is still used to derive "physically motivated" differences between NLSy1s and BLSy1s. Sources with broad-line widths larger than $2000 \mathrm{~km} \mathrm{~s}^{-1}$, but that have visible FeII emission and/or soft X-ray excess should also be considered as members of the NLSy1 group [33,14] (and refs. therein). To account for that, a luminositydependent definition of NLSy1 has been suggested several times (e.g. [21, 33, 10]). On the other hand, the broad line profiles are not all the same, one (or two) Gaussians (or Lorentzians) are required to model them $[33,23]$. The classification of Type 1s into population A or B sources that has a flexible limit at $\mathrm{FWHM}_{\mathrm{H} \beta \text {, broad }} \sim 4000 \mathrm{~km} \mathrm{~s}^{-1}$ - seems to account for this difference and for other observed properties [30]. Broad lines can also be narrowed due to the inclination of a ring-like broad line region [13]. However, the effects of the AGN orientation might not be equally important in all NLSy1s, as has been pointed out in particular sources [23, 8], and also using larger samples [5].

Emission-line ratios as indicators of the ionizing mechanism: $[\mathrm{OIII}] \lambda 5007 / \mathrm{H} \beta<3$. The denominator of this fraction refers to the total $\mathrm{H} \beta$ emission. In cases where the broad line region is obscured, like in Seyfert 2 galaxies, this ratio increases up to $\sim 5-10$. However, for Seyfert $1 \mathrm{~s}$ that do not suffer extinction ${ }^{2}$ it remains $\lesssim 3$ (e.g., [33]), and therefore it seems to be a condition of all unobscured Type $1 \mathrm{AGN}^{3}$. When comparing the [OIII] emission with the narrow $\mathrm{H} \beta$ component, NLSy1s span similar ranges as BLSy1s, but some objects also have $[\mathrm{OIII}] / \mathrm{H} \beta$ and $[\mathrm{NII}] / \mathrm{H} \alpha$ narrow-line ratios in the ranges of composite and star-forming galaxies (according to the classification by Kewley et al. 2001). Although it has been argued that this intrusion in the regions occupied by HII galaxies in the optical diagnostic diagrams might be an effect of the fitting procedure used to model the recombination lines ${ }^{4}$, it has been shown by independent studies that this

\footnotetext{
${ }^{1}$ Thought the most cited references are $[25,14]$ !

${ }^{2}$ Even in cases where the broad line region is obscured by the dust in the host galaxy - not that from the torus -, [OIII] $\lambda 5007 / \mathrm{H} \beta<3$, like in NGC 7172 [28].

${ }^{3}$ We verify this fact using the sample of Seyfert 1 s and QSOs of Dong et al. (2011). Out of 4178 objects only one, SDSS J015142.72+132003.3, has [OIII]/H $\beta>3(=4.29)$ [11]. Note that the sample selection in this case favored sources with minimal galaxy contamination. In other samples, where fitting the stellar component in the optical is required, this criterium is full filled by $\gtrsim 90 \%$ of the sources, as we corroborate using the measurements of Zhou et al. (2006) and Dong et al. (2012) [37, 12].

${ }^{4}$ Véron-Cetty et al. (2001) show that fitting the broad components with Lorentzians and the narrow ones with Gaussians in their sample of $\sim 60$ NLSy1s produce an overall shift in the distribution of these sources in the $[\mathrm{OIII}] / \mathrm{H} \beta$ vs. $[\mathrm{NII}] / \mathrm{H} \alpha$ diagram. In this way, only $\sim 10 \%$ instead of $\sim 30 \%$ of their sources are out of the Seyfert region.
} 

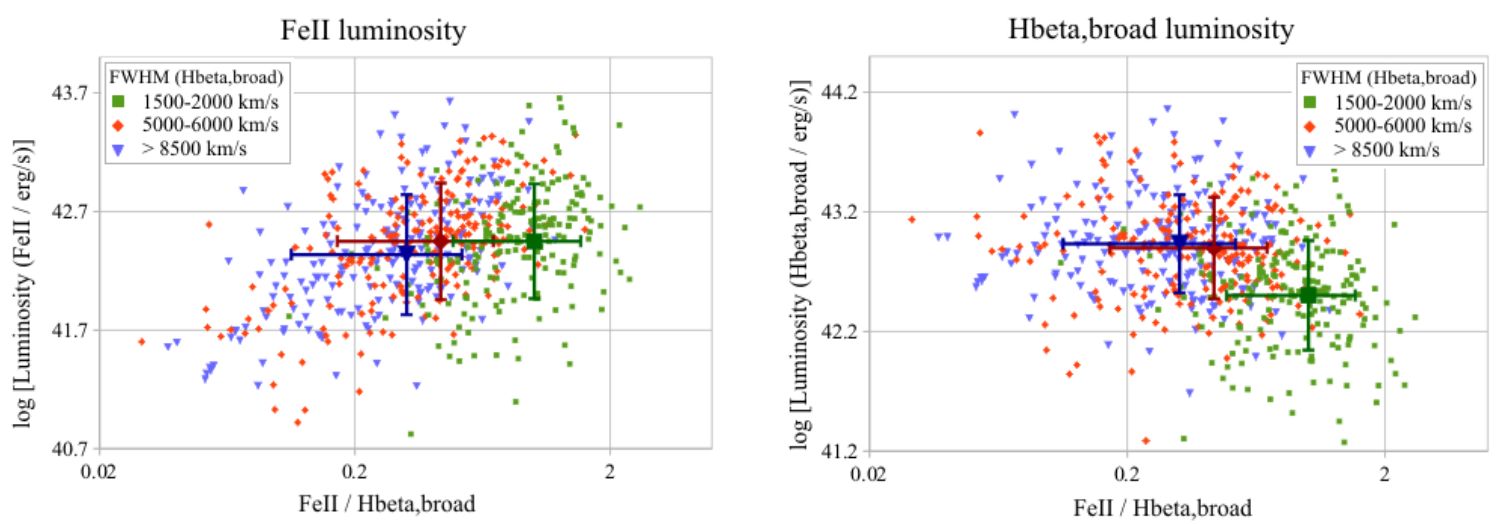

Figure 1: Iron and $\mathrm{H} \beta$, broad luminosities in Type 1 AGN. The symbols(colours) represent sources having $\mathrm{FWHM}_{\mathrm{H} \beta \text {, broad }}$ within certain ranges, as shown in the caption. Here, only 200 (randomly selected from the sample of Dong et al. 2011) objects from each group are shown. Big symbols and bars represent the mean and standar deviation of the distribution of luminosities and $R_{4570}$ for each group.

can also be a combined effect of the low- $M_{\mathrm{BH}}$ and dilution by the stellar component. For example, in the intermediate- $M_{\mathrm{BH}}$ sample of Xiao et al. (2011), 30\% of the objects lie under the Seyfert/starformation division [35]. The authors interpret this as a dilution effect because the median redshift of the HII sources is somewhat larger $(z \sim 0.1)$ than that of the whole sample $(z \sim 0.08)$. Interestingly, Stern \& Laor (2012) find that the fraction of Type 1 AGN classified as star forming sources (in the optical diagrams) increases when decreasing $M_{\mathrm{BH}}$ : from $6 \%$ at $\log \left(M_{\mathrm{BH}} / M_{\odot}\right)=8.8$ to $32 \%$ at $\log \left(M_{\mathrm{BH}} / M_{\odot}\right)=6.3$ (Fig. B1 in [29]).

Strong FeII optical emission. FeII multiplets are present in the spectra of Type 1 AGN from the UV to the infrared [7, 4]. The detection of optical FeII was the first property that allowed the identification of NLSy1 candidates among the Seyfert 2s in the available surveys[27]. The strength of the iron emission is usually quantified as the ratio $R_{4570}$ between the fluxes (or equivalent widths) of the FeII blend centered at $\lambda 4570$ and the $\mathrm{H} \beta$ broad component. NLSy1s were initially found to have $R_{4570} \gtrsim 0.5$. However, some research groups have pointed out that NLSy1s might not be strong FeII emitters, but faint $\mathrm{H} \beta$ sources ([33] and refs. therein). Using the measurements of Dong et al. $(2011)^{5}$ in a sample of $\sim 4000$ Type 1 AGN, we find that both statements are probably correct (see Fig. 1). We selected random subsamples of $\sim 200$ objects in three ranges of $\mathrm{FWHM}_{\mathrm{H} \beta \text {,broad }}$ : $1500-2000,5000-6000$, and $>8500 \mathrm{~km} \mathrm{~s}^{-1}$.

We found that, on average, the FeII luminosity stays approximately constant over the first two intervals $\left\langle\log \left(L_{\mathrm{FeII}} / \mathrm{erg} \mathrm{s}^{-1}\right)\right\rangle \sim 42.45$ and drops in the last one $(\sim 42.35)$, while the luminosity of the $\mathrm{H} \beta$ broad component is lower in the first group of sources $\left\langle\log \left(L_{\mathrm{H} \beta \text {, broad }} / \mathrm{erg} \mathrm{s}^{-1}\right)\right\rangle \sim 42.50$ than in the other two $(\sim 42.90)^{6}$. On the other hand, the large range of $R_{4570}$ seen in sources with

\footnotetext{
${ }^{5}$ In order to allow comparison with other samples, we use the total iron flux, i.e., we sum up the contributions of narrow and broad FeII fitted by the authors [11].

${ }^{6}$ It must be noticed that the measurement uncertainties of the FeII flux are $\sim 15 \%$ [11], which corresponds to $\sim 0.08$ dex. However, if the difference in $\left\langle L_{\mathrm{FeII}}\right\rangle$ between the three intervals were dominated by this kind of uncertainties, one would expect the average luminosity values to scatter around a mean value when one uses different subsamples of randomly selected 200 objects in each interval to estimate the average. In this case instead, we see a systematic shift in $\left\langle L_{\mathrm{FeII}}\right\rangle$ from the first two $\mathrm{FWHM}_{\mathrm{H} \beta \text {, broad }}$-bins to the last one. The measurement uncertainties in the $\mathrm{H} \beta$ broad-line
} 
low-FWHM $\mathrm{H}_{\mathrm{H} \text {, broad }}$ is due to the higher spread in the luminosity measurements of the $\mathrm{H} \beta$ broad component $(\sim 0.5 \mathrm{dex})$ compared to that in other Type $1 \mathrm{~s}(\sim 0.2 \mathrm{dex})-$ is this broad $R_{4570}$-range related to inclination effects? As a result, not all low-FWHM $\mathrm{H} \beta$, broad are high- $R_{4570}$ sources. In the NLSy1 sample of Zhou el at. (2006), the authors point out the presence of objects with very faint or non-detectable FeII emission, which they called FeII-deficient NLSy1s [37].

Steep soft $X$-ray spectrum (soft $X$-ray excess) and short-term $X$-ray variability. In the soft X-rays (i.e. below $\sim 1 \mathrm{keV}$ ), NLSy1s seem to be brighter and more variable than BLSy1s (e.g. $[30,33,21]$ and ref. therein). This has been associated with high Eddington ratios. However, not all NLSy1s behave the same. For example, Panessa et al. (2011) found that "hard [ $>20 \mathrm{keV}] \mathrm{X}$-ray selected NLSy1s do not display particularly strong soft excess emission"" [22]. Similar results had been reported by Williams et al. $(2002,2004)$. They noticed that a substantial number of optically bright, low-redshift NLSy1s did not have soft X-ray counterparts detected by ROSAT. Follow-up Chandra observations of 17 sources (with NLSy 1 characteristics, i.e. $\mathrm{FWHM}_{\mathrm{H} \beta \text {, broad }}<2000 \mathrm{~km} \mathrm{~s}^{-1}$ and $\left.R_{4570}>0.5\right)$ showed that their soft X-ray indices $(\Gamma=1.1-3.4)$ "extended far below those normally observed in NLSy1s"[38]. On the other hand, the extreme variability on short-time scales observed in the soft X-rays seems to be related to the comparatively low black hole masses found in these AGN rather than to the possibly high Eddington ratios [9, 1]. Ai et al. (2011) suggested the existence of two kinds of low- $M_{\mathrm{BH}}$ AGN: those NLSy1-like with strong FeII, soft X-ray excess and high- $L_{\mathrm{bol}} / L_{\mathrm{Edd}}$, and others more similar to BLSy1s, i.e., weak FeII and non-ubiquitous soft X-ray excess. However, in the NLSy1 sample of Zhou et al. (2006) about $15 \%$ of the objects have weak iron strength, but high Eddington ratio (i.e. $R_{4570}<0.5$ and $L_{\mathrm{bol}} / L_{\mathrm{Edd}}>0.5$ ). Another case is the source IRAS 01072+4954 with no detected optical FeII, but $L_{\mathrm{bol}} / L_{\mathrm{Edd}}>0.2$ [31].

\section{Do comparative studies suffer from selection biases?}

Here, we briefly describe how a sample selection that uses the width of the broad hydrogen lines as a criterium produces a bias in the physical properties of the selected AGN that affects the results of comparative studies between NLSy1s and BLSy1s.

Accepting that the broad line region is virialized [23] and that its radius depends on the optical continuum luminosity at $5100 \AA$ (after starlight correction) as $r_{\mathrm{BLR}} \propto L_{5100}^{\sim 0.5}$ [19], it is easy to find that $\left(M_{\mathrm{BH}} / M_{\odot}\right) \approx L_{\mathrm{bol}} / L_{\mathrm{Edd}}(f / 592.5)^{2}\left(\mathrm{FWHM}_{\mathrm{H} \beta \text {, broad }} / \mathrm{km} \mathrm{s}^{-1}\right)^{4}$, where $f$ is the scaling factor. Therefore, selecting sources with $\mathrm{FWHM}_{\mathrm{H} \beta \text {, broad }}<2000 \mathrm{~km} \mathrm{~s}^{-1}$ imposes a maximum limit on the black hole masses of $\sim 2.5 \times 10^{7} M_{\odot}$ when $L_{\mathrm{bol}} / L_{\mathrm{Edd}}=1$ and the typical value $f=0.75$ are assumed $^{8}$. An upper boundary on $M_{\mathrm{BH}}$ also implies an upper limit for the bolometric luminosity of $\sim(3-10) \times 10^{45} \mathrm{erg} \mathrm{s}^{-1}$ (even when allowing super-Eddington sources). At these luminosities, low and high Eddington-ratio AGN are detected in complete samples, i.e. depending on their $M_{\mathrm{BH}}$, both BLSy1s and NLSy1s are observed. However at $M_{\mathrm{BH}} \sim$ few $10^{6} M_{\odot}$, or correspondingly $L_{\mathrm{bol}} \sim 10^{44} \mathrm{erg} \mathrm{s}^{-1}$, observational biases only favor the detection of high Eddington sources. While

fluxes are only $\sim 8 \%[11]$.

${ }^{7}$ The authors also pointed out that “...indeed only one source [out of 14], IGRJ $19378-0617$, shows a dominant and strongly variable soft-X-ray component".

${ }^{8}$ Correcting by the broad line profile (Gaussian or Lorentzian) can result in one order of magnitude higher black hole masses. See [8] for details. 
this kind of selection bias is extensively discussed by Hopkins et al. (2009) [18], there are indications that the dilution effects on low- $M_{\mathrm{BH}}$ sources might be even more severe particularly at optical/infrared bands - i.e. where most of the AGN with narrow broad components are identified - than it is estimated there. If the $M_{\mathrm{BH}}$ vs. bulge-mass relation is not a single power law across the whole black hole mass range, but is steeper at low black hole masses - as it has been suggested and observed in samples of nearby objects [20,16] -, then dilution effects become $10-100$ times more severe at $10^{6}-10^{5} M_{\odot}$ than previously estimated ${ }^{9}$. Hopkins et al. also show that samples with an implicit high Eddington-ratio selection -like those that are produced when selecting only narrow $\mathrm{H} \beta$ broad-line emitters - favor low-mass disk-dominated host galaxies, as has been found in the case of NLSy1s.

As an example, we compiled information about the initial group of NLSy1 sources studied by Osterbrock \& Pogge (1985) [25]. We noticed that all of them have relatively low black hole masses $\log \left(M_{\mathrm{BH}} / M_{\odot}\right) \sim 5.8-7.2$, and that most of them $(9 / 11)^{10}$ also have high Eddington ratios $L_{\mathrm{bol}} / L_{\mathrm{Edd}}>0.4$. As it was mentioned before, also the calibration and fitting procedures, even when using data from the same survey, can introduce biases in the sample selection. For example, the very careful selection process of low- $M_{\mathrm{BH}}$ sources applied by Dong et al. (2012) allowed them to detect AGNs with lower Eddington ratios $\left(\left\langle L_{\mathrm{bol}} / L_{\mathrm{Edd}} \sim\right\rangle-0.7\right.$ versus -0.4$)$ than previously published samples with similar selection criteria [12].

\section{Final comments}

Narrow Line Seyfert 1 have been considered a special class of AGN because of their apparently extreme properties. After an extensive literature review (not covered in the References of this short paper due to space restrictions) and studying some statistical properties of recently published samples of Type 1, NLSy1s, and low- $M_{\mathrm{BH}}$ sources $[11,37,12]$, we find that apart from the width of the broad $\mathrm{H} \beta$ component, which is an arbitrarily defined limit, none of the other properties is unique, nor ubiquitous of the NLSy1s. Moreover, these sources seem to be normal Type 1 AGN that naturally cover the lower end of the $\mathrm{FWHM}_{\mathrm{H} \beta \text {, broad }}$ range.

Implicit selection effects $\left(\mathrm{FWHM}_{\mathrm{H} \beta \text {, broad }}<2000 \mathrm{~km} \mathrm{~s}^{-1}\right)$ in NLSy1s samples can account at least partially - for the following averaged observed properties when they are analyzed in comparison to those of BLSy1s: lower $M_{\mathrm{BH}}$, faster soft X-ray variability, higher $L_{\mathrm{bol}} / L_{\mathrm{Edd}}$, lower $L_{\mathrm{bol}}$, higher bolometric corrections, lower redshifts, bluer disk-dominated hosts, $M_{\mathrm{BH}}$ below the bulge mass (or bulge luminosity) correlations and thereby higher importance of secular evolution processes.

On the other hand, it is possible to define a 'special' subgroup of sources that meet a certain set of observational requirements (in $\mathrm{FWHM}_{\mathrm{H} \text {,broad }}, R_{4570}, \Gamma, L_{\mathrm{bol}}$-correction, radio-loudness, nar-

\footnotetext{
${ }^{9}$ The dilution of the AGN signatures by the stellar emission of the host galaxy can be estimated as the contrast between the AGN $\left(L_{\mathrm{AGN}, \mathrm{B}} \sim L_{\mathrm{bol}} / 9\right)$ and the host luminosities, in the B-band $L_{\mathrm{AGN}, \mathrm{B}} / L_{\mathrm{host}, \mathrm{B}}=\frac{1}{3} \times$ $10^{3}\left(\lambda_{\mathrm{Edd}} / 0.1\right)\left(M_{\mathrm{BH}} / M_{\odot}\right)\left(L_{\mathrm{host}, \mathrm{B}} / L_{\odot, \mathrm{B}}\right)$, with $\lambda_{\mathrm{Edd}} \equiv L_{\mathrm{bol}} / L_{\mathrm{Edd}}$. The relation between the black hole and the bulge

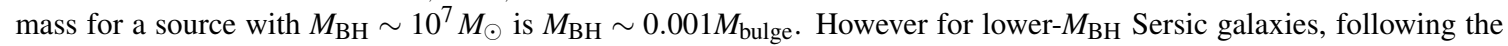
relation found by Scott et al. (2013), at $M_{\mathrm{BH}}=10^{6} M_{\odot}, M_{\mathrm{BH}} \sim 10^{-4} M_{\text {bulge }}$ [16].

${ }^{10}$ The other two sources: Mrk 1388 seems to be a high-ionization Seyfert 2 galaxy [26, 15], and Mrk 684 presents broad $\mathrm{H} \beta$ and strong FeII emission, but its Eddington ratio $L_{\mathrm{bol}} / L_{\mathrm{Edd}} \sim 10^{-7}$ is very unusual for a Type 1 source $\left(M_{\mathrm{BH}}\right.$ and $L_{\text {bol }}$ from [34, 17], see spectrum in [32]).
} 
row line ratios, host galaxy morphology...), but that might be of little physical interest. Selecting samples based on physical properties would be probably more helpful to understand the accretion phenomena. In the words of Goodrich (1989) ${ }^{11}$ "...a taxonomic system, based not necessarily on the most easily observed parameters but rather on the most physically relevant quantities and some theoretical picture, is required in order to make substantial progress in our interpretation of AGN" [14].

\section{References}

[1] Ai, Y.L. et al. 2011 ApJ 72731

[2] Antonucci, R. 1993 AR\&A 31473

[3] Antonucci, R. 2012 A\&AT 27557

[4] Rodríguez-Ardila, A.; Contini, M. \& Viegas, S.M. 2005 MNRAS 357

[5] Boroson, T.A. 2011 in PoS(NLS1)003

[6] Boroson, T.A. 2002 ApJ 56578

[7] Boroson, T.A. \& Green, R.F. 1992 ApJS 80109

[8] Collin, S. et al. 2006 A\&A 45675

[9] Dewangan G.C. et al. 2008 ApJ 689762

[10] Dultzin, D et al. 2011 in PoS(NLS1)12

[11] Dong, X.-B. et al. 2011 ApJ 73686

[12] Dong, X.-B. et al. 2012 ApJ 755167

[13] Gaskell, C.M. 2009 NewARev 53140

[14] Goodrich, R.W. 1989 ApJ 342224

[15] Gordon, M. 1988 PASP 100623

[16] Scott, N.; Graham, A. \& Schombert, J. 2013 ApJ 76876

[17] Grupe, D. et al. 2004 ApJ 127156

[18] Hopkins, P.F. et al. 2009 MNRAS 398333

[19] Kaspi, S. et al. 2000 ApJ 533631

[20] Laor, A. 2001 ApJ 533677

[21] Laor, A. 2000 NewARev 44503

[22] Panessa, F. et al. 2011 MNRAS 4172426

[23] Peterson, B.M. 2012 in PoS(NLS1)032

[24] Popovic, L.C.; Kovacevic, J. \& Dimitrijevic, M.S. 2013 (this proceedings)

[25] Osterbrock, D.E. \& Pogge, R.W. 1985 ApJ 297166

[26] Osterbrock, D.E. 1985 PASP 9725

\footnotetext{
${ }^{11}$ This is Goodrich's reading of the review made by Lawrence (1987) on the AGN classification and its meaning.
} 
[27] Osterbrock, D.E. \& Pogge, R.W. 1987 ApJ 323108

[28] Smajic, S. et al. 2012 A\&A 544105

[29] Stern, J. \& Laor, A. 2012 MNRAS 431836

[30] Sulentic, J.W. et al. 2000 ApJ 536 L5

[31] Valencia-S., M. et al. 2012 A\&A 544129

[32] Vaughan, S. et al. 2001 MNRAS 327673

[33] Véron-Cetty, M.-P.; Véron, P. \& Goncalves, A.C. 2001 A\&A 372730

[34] Wang, T. \& Lu, Y. 2001 A\&A 37752

[35] Xiao, T. et al. 2011 ApJ 73928

[36] Xu, D. 2012 AJ 14383

[37] Zhou, H. et al. 2006 ApJSS 166128

[38] Williams, R.J.; Mathur, S. \& Pogge, R.W. 2004 ApJ 610737 